\title{
ISOTROPY GROUPS OF HOMOTOPY CLASSES OF MAPS
}

\author{
G. TRIANTAFILLOU
}

\begin{abstract}
Let aut $(X)$ be the group of homotopy classes of self-homotopy equivalences of a space $X$ and let $[f] \in[X, Y]$ be a homotopy class of maps from $X$ to $Y$. The aim of this paper is to prove that under certain nilpotency and finiteness conditions the isotropy group $\operatorname{aut}(X)_{[f]}$ of $[f]$ under the action of $\operatorname{aut}(X)$ on $[X, Y]$ is commensurable to an arithmetic group. Therefore aut $(X)_{[f]}$ is a finitely presented group by a result of Borel and Harish-Chandra.
\end{abstract}

\section{INTRODUCTION}

Throughout this paper we consider spaces with base points and based homotopy classes of maps.

We reall that a group $G$ is nilpotent if its lower central series $\Gamma^{1}(G)=G$, $\Gamma^{i+1}(G)=\left[G, \Gamma^{i} G\right]$, reaches $\{1\}$ in a finite number of steps. Let $G$ act from the left on an abelian group $A$ by automorphisms. Let $\Gamma^{1}(A)=A$ and let $\Gamma^{i+1}(A)$ be the subgroup of $A$ generated by $\left\{x a-a, x \in G, a \in \Gamma^{i}(A)\right\}$. We say that $K$ operates nilpotently on $A$ if $\Gamma^{i}(A)=\{0\}$ for some $i$.

Definition 1.1. A space $X$ is said to be nilpotent if $\pi_{1}(X)$ is nilpotent and acts nilpotently on the higher homotopy groups of $X$.

Let $X_{0}$ denote the rationalization of $X$ and let $f_{0}: X_{0} \rightarrow Y_{0}$ denote the rationalization of a map $f: X \rightarrow Y$ [S1, B-K, Q].

Theorem 1.2. Let $X$ be a nilpotent finite $C W$-complex and let $Y$ be a nilpotent $C W$-complex such that each $\pi_{i}(Y)$ is finitely generated. Let $[f] \in[X, Y]$ be a homotopy class of maps. Then

(i) The isotropy group aut $\left(X_{0}\right)_{\left[f_{0}\right]}$ of $\left[f_{0}\right] \in\left[X_{0}, Y_{0}\right]$ under the action of aut $\left(X_{0}\right)$ is a linear algebraic group over $\mathbf{Q}$.

(ii) The isotropy group aut $(X)_{[f]}$ of $[f]$ under the action of aut $(X)$ is commensurable with an arithmetic subgroup of $\operatorname{aut}\left(X_{0}\right)_{\left[f_{0}\right]}$. Hence aut $(X)_{[f]}$ is finitely presented.

Definition 1.3. A group $G$ is said to be of finite type if the space $K(G, 1)$ has the homotopy type of a $C W$-complex with a finite number of cells in each dimension.

Received by the editors March 19, 1990.

1980 Mathematics Subject Classification (1985 Revision). Primary 55P10, 55P62; Secondary 20G99, 10E45.

Key words and phrases. Homotopy self-homotopy equivalence, arithmetic group, minimal model. 
Remark 1. The notion of a group being of finite type is in general stronger than being finitely presented although for nilpotent groups the two notions coincide.

By a result of Borel-Serre [B-S] every arithmetic subgroup of an algebraic group is of finite type. Applying this and a fibration argument of [D-D-K] to Theorem 1.2 yields

Corollary 1.5. The isotropy group $\operatorname{aut}(X)_{[f]}$ is of finite type.

In the case when $f$ is a constant map and $\operatorname{aut}(X)_{[f]}=\operatorname{aut}(X)$, the result is due to Wilkerson [W] (for simply connected $X$ ) and to Sullivan [S2] (for nilpotent $X)$. The case was further extended to virtually nilpotent spaces $X$ by Dror, Dwyer and Kan [D-D-K].

The equivariant version of the theorem is going to be part of my joint work with Mel Rothenberg On the classification of G-manifolds up to finite ambiguity, where it is applied to prove the classification result. Other applications, in particular to surgery theory may be possible in view of the action of $\operatorname{aut}(X)$ on the set of normal maps $[X, G /$ Cat $]$, where Cat $=0$, PL or Top.

The proof of the arithmeticity of $\operatorname{aut}(X)$ is based either on the minimal model theory [S2] or on the theory of simplicial groups [W, D-D-K]. The methods based on the latter theory seem to get very complicated when applied to study the action of aut $(X)$ on $[X, Y]$. An attempt has been made by S. Hurwitz $[\mathrm{Hr}]$ to obtain similar results for fibrations under more restricted hypotheses by using the simplicial method. However, there is a serious gap in the argument of that paper. We will present here a proof of the arithmeticity of $\operatorname{aut}(X)_{[f]}$ based on Sullivan's theory of minimal models. Since the rational homotopy theory of nilpotent $C W$-complexes is equivalent to the homotopy theory of minimal nilpotent differential graded algebras (dga) over $\mathbf{Q}$, we replace $\left[X_{0}, Y_{0}\right]$ with $[\mathscr{M}, \mathscr{N}]$; here $\mathscr{M}$ and $\mathscr{N}$ are the minimal models of $Y$ and $X$ respectively, and $[\mathscr{M}, \mathscr{N}]$ means homotopy classes of dga-maps. Let $\operatorname{aut}(\mathcal{N})$ denote the group of homotopy classes of automorphisms of $\mathscr{N}$ and let $\operatorname{aut}(\mathscr{N})_{[\varphi]}$ denote the isotropy group of $[\varphi] \in[\mathscr{M}, \mathscr{N}]$. In order to prove that $\operatorname{aut}(\mathscr{N})_{[\varphi]}$ is an algebraic group we want to compare aut $(\mathscr{N})_{[\varphi]}$ to a group of actual automorphisms rather than homotopy classes of automorphisms. Finding such a group of actual automorphisms presents the heart of the argument. $\operatorname{Aut}(\mathcal{N})_{\varphi}$, i.e. the group of automorphisms of $\mathcal{N}$ which preserve $\varphi$, is not a good choice since these automorphisms do not represent all homotopy classes in $\operatorname{aut}(\mathscr{N})_{[\varphi]}$. It turns out that if we replace $\varphi: \mathscr{M} \rightarrow \mathscr{N}$ by its so-called minimal extension $i: \mathscr{M} \hookrightarrow \mathscr{A}$, then $\operatorname{Aut}(\mathscr{A})_{i}$ has the desired properties. Namely, $\operatorname{Aut}(\mathscr{A})_{i}$ is a linear algebraic group over $\mathbf{Q}$ and there is a group epimorphism

$$
\operatorname{Aut}(\mathscr{A})_{i} \rightarrow \operatorname{aut}(\mathscr{N})_{[\varphi]}
$$

with algebraic kernel. This provides $\operatorname{aut}(\mathcal{N})_{[\varphi]}$ with an algebraic group structure.

In order to prove the second statement of Theorem 1.2, we use an inductive argument over the Postnikov tower of principal fibrations of $Y$. The group $\operatorname{aut}(\mathcal{N})_{[\varphi]}$ turns out to be the limit of isotropy groups in a sequence of algebraic actions on vector spaces over $\mathbf{Q}$, namely on certain quotients of cohomology groups with coefficients in $\mathbf{Q}$. The main difficulty is to show that the actions are indeed algebraic. Again the notion of minimal extension of a dga map is crucial in the construction of these algebraic actions. 
I would like to thank Mel Rothenberg and Tyakal Venkataramana for helpful discussions.

In the next section we give some preliminaries on the theory of differential graded algebras and on the theory of algebraic groups.

\section{Preliminaries}

For any simplicial complex $X$ or more generally any semisimplicial set $X$ Sullivan defined certain rational P.L. forms on $X$ which form a differential graded-commutative algebra $A(X)$ over $\mathbf{Q} . A(X)$ is called the de Rham complex of $X$ and provides the connection between spaces and dga's. Here we recall some definitions and results about dga's which will be used in the proof of Theorem 1.2. This material can be found in [S2, H, G-M and B-G].

We consider augmented dga's $\varepsilon: \mathscr{A} \rightarrow \mathbf{Q}$ and denote by $\mathscr{A}^{+}=\operatorname{ker} \varepsilon$. We further assume that all dga's $\mathscr{A}$ in sight are connected, i.e., $H^{0}(\mathscr{A})=\mathbf{Q}$, and that they have finitely generated cohomology in each dimension.

Let $V=\bigoplus_{n \geq 0} V_{n}$ be a graded vector space. By $\Lambda V$ we denote the free graded-commutative algebra generated by $V . \Lambda V_{n}$ is the exterior algebra on $V_{n}$ if $n$ is odd and it is the polynomial algebra on $V_{n}$ if $n$ is even. $\Lambda V=$ $\bigotimes_{n \geq 0} \Lambda V_{n}$.

Definition 2.1. A dga is called free if it is free as a commutative algebra.

Definition 2.2. A dga $\mathscr{M}$ is called minimal if (a) $\mathscr{M}$ is free and (b) $d \mathscr{M} \subseteq$ $\mathscr{M}^{+} \cdot \mathscr{M}^{+}$.

Definition 2.3. A Hirsch extension or elementary extension $\mathscr{A} \otimes_{d} \Lambda V$ of a dga $\mathscr{A}$ is defined as follows: Let $V$ be a finite dimensional vector space homogeneous of degree $k$, and let $d$ be a linear map $d: V \rightarrow Z^{k+1}(\mathscr{A})$, where $Z^{k+1}(\mathscr{A})$ is the space of cocycles of $\mathscr{A}$ of degree $k+1 . \mathscr{A} \otimes_{d} \Lambda V$ is equal to $\mathscr{A} \otimes \Lambda V$ as an algebra and its differential is determined by the differential of $\mathscr{A}$ and $d: V \rightarrow Z^{k+1}(\mathscr{A})$. Changing $d: V \rightarrow Z^{k+1}(\mathscr{A})$ by coboundaries yields isomorphic Hirsch extensions. Hirsch extensions correspond to principal fibrations in a way to be described below.

Definition 2.4. Let $\mathscr{M}$ be a minimal algebra and let $\mathscr{M}(n)$ be its subalgebra generated by elements of degree $\leq n$. We say that $\mathscr{M}$ is nilpotent if each $\mathscr{M}(n)$ results from $\mathscr{M}(n-1)$ by a finite sequence of elementary extensions.

We consider the $(t, d t)$-notion of homotopy between dga maps as developed in [G-M]. Let $\Lambda(t, d t)$ denote the free dga on generators $t$ and $d t$ of degree 0 and 1 respectively with $d(t)=d t$.

Definition 2.5. Two dga maps $\varphi, \psi: \mathscr{A} \rightarrow \mathscr{B}$ are said to be homotopic if there is a dga map $H: \mathscr{A} \rightarrow \mathscr{B} \otimes \Lambda(t, d t)$ such that $\left.H\right|_{t=0, d t=0}=\varphi$ and $\left.H\right|_{t=1, d t=0}=\psi$.

The basic fact about Hirsch extensions is the homotopy extension property. We are going to need a special case of it. Namely

Proposition 2.6 [G-M]. Given a diagram

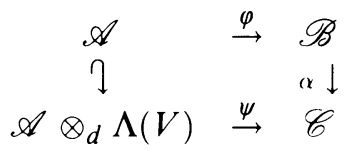


where $\alpha$ is a cohomology isomorphism and given a homotopy $H: \mathscr{A} \rightarrow \mathscr{C} \otimes$ $\Lambda(t, d t)$ from $\alpha \circ \varphi$ to $\psi \mid \mathscr{A}$, there is an extension $\tilde{\varphi}: \mathscr{A} \otimes_{d} \Lambda(\Lambda) \rightarrow \mathscr{B}$ of $\varphi$ and an extension of $H$ to a homotopy from $\alpha \circ \tilde{\varphi}$ to $\psi$.

We have the following properties of minimal dga's.

Theorem 2.7 [S2]. For every dga $\mathscr{A}$ there is a minimal dga $\mathscr{M}$ and a dga map $\rho: \mathscr{M} \rightarrow \mathscr{A}$ which is an isomorphism on cohomology. If there are two minimal algebras $\rho: \mathscr{M} \rightarrow \mathscr{A}$ and $\rho^{\prime}: \mathscr{M}^{\prime} \rightarrow \mathscr{A}$ with this property, there is an isomorphism $\alpha: \mathscr{M} \rightarrow \mathscr{M}^{\prime}$ such that $\rho^{\prime} \alpha \simeq \rho$.

Definition 2.8. $\mathscr{M}$ is then called the minimal model of $\mathscr{A}$.

Definition 2.9. A free dga $\Lambda V$ is said to be acyclic if $V=U \oplus d U$, i.e., $V$ splits up into a direct sum of two isomorphic vector spaces $U$ and $d U$ and $d(U)=d U$.

Theorem 2.10 [S2]. If $\mathscr{A}$ is a free dga then $\mathscr{A}$ is isomorphic to the product $\mathscr{A}=\mathscr{M} \otimes \Lambda(V)$ of two dga's, where $\mathscr{M}$ is a minimal dga and $\Lambda(V)$ is a free acyclic dga.

We give now some further notions of extensions of dga's which will serve as models for fibrations of spaces. Let $\mathscr{A}$ be a dga.

Definition 2.11. A dga $\mathscr{B}$ is called a contractible or acyclic extension of $\mathscr{A}$ if $\mathscr{B}=\mathscr{A} \otimes \Lambda V$, where $\Lambda V$ is a free acyclic dga.

Definition 2.12. A dga $\mathscr{B}$ is called a minimal extension of $\mathscr{A}$ if $\mathscr{B}$ results from $\mathscr{A}$ by a finite sequence of Hirsch extensions, i.e., $\mathscr{B}=\mathscr{A} \otimes \Lambda\left(\bigoplus_{i=1}^{k} V_{i}\right)$ as algebras and the differential of $\mathscr{B}$ is determined by the differential of $\mathscr{A}$ and differentials $d \mid V_{m}: V_{m} \rightarrow \mathscr{A} \otimes \Lambda\left(\bigoplus_{i=1}^{m-1} V_{i}\right)$.

Definition 2.13. A dga $\mathscr{B}$ is called a free extension or $K S$-extension (cf. [H]) of $\mathscr{A}$ if $\mathscr{B}$ results from $\mathscr{A}$ by a finite sequence of Hirsch or acyclic extensions.

Remark 2.14. We recall that we consider augmented dga's and maps that preserve the augmentation. In the above extensions consider the ideal $I(\mathscr{A})$ in $\mathscr{B}$ generated by $\mathscr{A}$, and form the quotient $\mathscr{B} / I(\mathscr{A})$. The quotient is a dga which as an algebra is equal to $\Lambda V$.

Proposition $2.15[\mathrm{H}]$. An extension $\mathscr{B}$ of $\mathscr{A}$ is acyclic, minimal or free if and only if $\mathscr{B} / I(\mathscr{A})=\Lambda V$ is an acyclic, minimal or free dga respectively.

Remark 2.16. The free extensions are the "cofibrations" and the free dga's are the "cofibrant" objects in the category of dga's.

We have now the following result about cohomology isomorphisms between minimal extensions.

Theorem 2.17. Let $\mathscr{B}$ and $\mathscr{B}^{\prime}$ be minimal extensions of $\mathscr{A}$ and $\mathscr{A}^{\prime}$ respectively. Let $(\varphi, \psi)$ be a map of extensions; i.e., a pair of maps such that the diagram

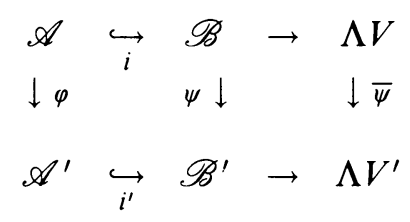


commutes, where $\bar{\psi}$ is the induced quotient map. If $\varphi$ and $\psi$ are cohomology isomorphisms, then $\bar{\psi}$ is an isomorphism between the quotient minimal dga's $\Lambda V$ and $\Lambda V^{\prime}$. Moreover, if $\varphi$ is an isomorphism and $\psi$ is a cohomology isomorphism then $\psi$ is an isomorphism $[\mathrm{H}, p$. 54].

The importance of minimal extensions is that they can serve as models for arbitrary dga maps in the following sense.

Theorem $2.19[\mathrm{H}]$. Let $\varphi: \mathscr{A} \rightarrow \mathscr{C}$ be a dga map. Then there is a minimal extension $i: \mathscr{A} \mapsto \mathscr{B}$ and a dga map $\sigma: \mathscr{B} \rightarrow \mathscr{C}$ such that

(i) $\varphi=\sigma \circ i$ and

(ii) $\sigma$ is a cohomology isomorphism.

Now let $X$ be a connected space of the homotopy type of a CW-complex with a base point, and let $S(X)$ be the singular complex of $X$. Let $A(X)$ be the de Rham complex of rational PL forms of $S(X) . A(X)$ is a connected augmented dga. Let $\mathscr{M}$ be the minimal model of $A(X)$. Then $\mathscr{M}$ is called the minimal model of the space $X$. If $X$ is a nilpotent space then $\mathscr{M}$ is a nilpotent algebra, and the decomposition of $\mathscr{M}$ into a union of Hirsch extensions corresponds to the decomposition of the space $X$ into its Postnikov tower of principal fibrations. The precise correspondence is as follows:

We consider a principal fibration $F \rightarrow E \stackrel{p}{\rightarrow} B$, where $F$ is an EilenbergMac Lane space $K(\pi, n)$ and $\pi_{1}(B)$ acts trivially on the fiber up to homotopy. Let $k \in H^{n+1}(B ; \pi)$ be the cohomology class that classifies $p$ and let $k_{0}$ be its image in $H^{n+1}(B, \pi \otimes \mathbf{Q})$. Let $\rho: \mathscr{M} \rightarrow A(B)$ be a minimal model for the base space $B$. The map $\rho$ induces an isomorphism

$$
H^{n+1}\left(\mathscr{M}, \pi^{*}\right) \cong H^{n+1}(B ; \pi \otimes \mathbf{Q}),
$$

where $\pi^{*}=\operatorname{Hom}(\pi, \mathbf{Q})$; here the cohomology of a dga $\mathscr{M}$ with coefficients in a vector space $V$ is defined as $H^{*}(\mathscr{M}, V) \equiv H^{*}(\operatorname{Hom}(V, \mathscr{M}))$.

We take $[d] \in H^{n+1}\left(\mathscr{M}, \pi^{*}\right)$ the cohomology class which corresponds to $k_{0}$ and choose a representative $d: \pi^{*} \rightarrow Z^{n+1}(\mathscr{M})$. We can construct a Hirsch extension $\mathscr{M} \otimes_{d} \Lambda\left(\pi^{*}\right)$, which depends only on [d] up to isomorphism. It turns out that this dga is the minimal model of the total space $E$.

Theorem 2.20 [G-M, p. 133 and H, p. 257]. There is a cohomology isomorphism $\tilde{\rho}: \mathscr{M} \otimes_{d} \Lambda\left(\pi^{*}\right) \rightarrow A(E)$ such that the diagram

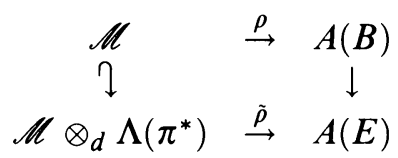

commutes. This Hirsch extension is the only one up to isomorphism that admits such a map $\tilde{\rho}$.

Conversely, let $\mathscr{M} \otimes_{d} \Lambda V$ be a Hirsch extension and let $\rho: \mathscr{M} \rightarrow A(B)$ be the minimal model of a space $B$. Then there is a principal fibration $F \rightarrow$ $E \rightarrow B$ over $B$ with fiber $F=K\left(V^{*}, n\right)$ and a cohomology isomorphism $\tilde{\rho}: \mathscr{M} \otimes_{d} \Lambda V \rightarrow A(E)$ such that the same diagram as above commutes. This fibration is unique up to equivalence.

In the rest of this section we give some definitions and facts from the theory of algebraic groups that are needed in the proofs. This material can be found in [B-HC]. 
Let $V$ be an $n$-dimensional vector space over $\mathbf{Q}$, and let $\mathrm{GL}(V)$ denote the group of linear automorphisms of $V$. Consider the elements of $\mathrm{GL}(V)$ as matrices with entries in $\mathbf{Q}$.

Definition 2.21. A subgroup $G \subseteq \mathrm{GL}(V)$ is called a linear algebraic group over $\mathbf{Q}$ or an algebraic matrix group over $\mathbf{Q}$ if it is the annihilator of some set of polynomials on the entries with rational coefficients.

Remark. $\mathrm{GL}(V)$ can be considered as an algebraic subgroup of $\mathrm{GL}(V \oplus \mathbf{Q})$ containing the matrices of the form $\left(\begin{array}{ll}g & 0 \\ 0 & x\end{array}\right)$ which satisfy $x \cdot \operatorname{det} g=1$, where $g \in \mathrm{GL}(V)$.

Definition 2.22. A group homomorphism $f: G \rightarrow G^{\prime}$ between two linear algebraic groups is said to be algebraic if the entries of $f(g)$ are polynomial functions of the entries of $g \in G$.

Let $G \subset \mathrm{GL}(V)$ be a linear algebraic group and let $L \subset V$ be a lattice in $V$, i.e., a finitely generated subgroup of maximal rank. Let $G(L)$ denote the subgroup of the automorphisms $g \in G$ such that $g(L)=L$. A different choice of lattice $L^{\prime}$ leads to a commensurable subgroup $G\left(L^{\prime}\right)$, i.e. $G(L) \cap G\left(L^{\prime}\right)$ is a subgroup of finite index in both $G(L)$ and $G\left(L^{\prime}\right)$.

Definition 2.23. A subgroup of a linear algebraic group $G$ is called an arithmetic subgroup of $G$ if it is commensurable to $G(L)$ for some lattice $L \subset G$.

\section{Proofs}

Proof of Theorem 1.2(i). Let $\rho: \mathscr{M} \rightarrow A(Y) \rightarrow A\left(Y_{0}\right)$ and $\sigma: \mathscr{N} \rightarrow A(X) \rightarrow$ $\mathscr{A}\left(X_{0}\right)$ be minimal models of $Y$ and $X$ (or $Y_{0}$ and $X_{0}$ ) respectively. Then $[f] \in[X, Y]$ induces a unique homotopy class $[\varphi] \in[\mathscr{M}, \mathscr{N}]$ by [G-M, p. $181]$, such that the diagram

$$
\begin{array}{ccc}
A(Y) & \vec{f} & A(X) \\
\uparrow \rho & & \uparrow \sigma \\
\mathscr{M} & \vec{\varphi} & \mathscr{N}
\end{array}
$$

commutes up to homotopy. We consider the action of aut $(\mathscr{N})$ on $[\mathscr{M}, \mathscr{N}]$ by composition and we will show that $\operatorname{aut}(\mathscr{N})_{[\varphi]}$ is an algebraic subgroup of aut $(\mathscr{N})$.

We consider a minimal extension $i: \mathscr{M} \mapsto \mathscr{A}$ for $\varphi$ and a cohomology isomorphism $\pi: \mathscr{A} \rightarrow \mathscr{N}$ such that $\varphi=\pi \circ i$;

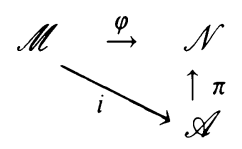

We establish some notation:

$\operatorname{Aut}(\mathscr{A})$ is the group of dga automorphisms (isomorphisms) of $\mathscr{A}$.

aut $(\mathscr{A})$ is the group of homotopy classes of self-homotopy equivalences of $\mathscr{A}$.

$\operatorname{Aut}(\mathscr{A}, \operatorname{rel} \mathscr{M})$ is the subgroup of $\operatorname{Aut}(\mathscr{A})$ containing isomorphisms $\alpha: \mathscr{A}$ $\rightarrow \mathscr{A}$ such that $\alpha \mid \mathscr{M}=\mathrm{id}_{\mathscr{M}}$.

$\operatorname{aut}(\mathscr{A}, \widetilde{\operatorname{rel}} \mathscr{M})$ is the subgroup of aut $(\mathscr{A})$ containing the elements $[\alpha]$ with the property $\alpha \mid \mathscr{M} \simeq i: \mathscr{M} \mapsto \mathscr{A}$. 
$\operatorname{aut}(\mathscr{N})_{[\varphi]}$ is the subgroup of $\operatorname{aut}(\mathscr{N})$ containing the elements $[b]$ such that $b \circ \varphi \simeq \varphi$.

$\operatorname{End}(\mathscr{N}, \mathscr{A})$ is the set of injective dga maps from $\mathscr{N}$ to $\mathscr{A}$.

We have the following commutative diagram

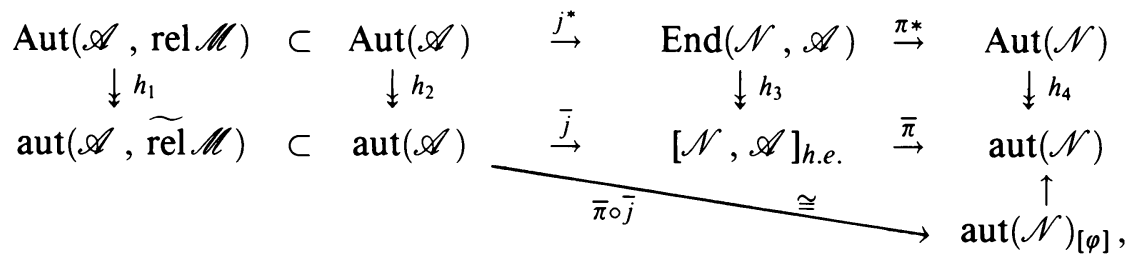

where the groups of the second row are quotients of the sets of the first row modulo homotopy respectively. In order to explain the maps $\pi_{*}$ and $j^{*}$ we note the following: Since $\mathscr{M}$ is free, $\mathscr{A}$ is also free by construction. Therefore, $\mathscr{A}$ can be written as $\mathscr{A}=\mathscr{N}^{\prime} \otimes \Lambda(U \oplus d U)$ by Theorem 2.10 , where $\mathscr{N}^{\prime}$ is a minimal dga. The composition $\mathscr{N}^{\prime} \mapsto \mathscr{A} \stackrel{\pi}{\rightarrow} \mathscr{N}$ is a cohomology isomorphism between minimal dga's and therefore it is an isomorphism by [S2]. It follows that $\pi$ is surjective, and there is a splitting $j: \mathscr{N} \rightarrow \mathscr{A}$ such that $\pi \circ j=\mathrm{id}_{\mathscr{N}}$. Moreover, we can choose $U$ and $d U$ on the level of the indecomposables of $\mathscr{A}$ in such a way that the kernel of $\pi$ is the ideal generated by the acyclic subalgebra $\Lambda(U \oplus d U)$. Hence we can identify $\mathscr{A}=\mathscr{N} \otimes \Lambda(U \oplus d U)$ as a tensor product of dga's. We define $\pi_{*}$ to be composition by $\pi$, i.e., $b \in \operatorname{End}(\mathscr{N}, \mathscr{A})$ is sent to its projection $\pi \circ b: \mathscr{N} \rightarrow \mathscr{N}$. From the above argument follows that $\pi \circ b$ is an isomorphism. We also define $j^{*}(\alpha)=\alpha \circ j$, the restriction of $\alpha$ to the subalgebra $\mathscr{N} \subset \mathscr{A}$. The map $j^{*}$ is surjective: Let $\beta: \mathscr{N} \rightarrow \mathscr{A}$ be an injective map of dga's. Define $\alpha: \mathscr{A} \rightarrow \mathscr{A}$ to be equal to $\beta$ when restricted to $\mathcal{N}$ and equal to id $\mid \Lambda(U \oplus d U)$ when restricted to $\Lambda(U \oplus d U)$. Then $\alpha$ is an isomorphism because it induces an isomorphism on the indecomposables. The maps $\bar{j}$ and $\bar{\pi}$ are induced by $j^{*}$ and $\pi_{*}$ respectively.

The map $h_{3}$ is a surjective map onto the set of homotopy classes of homotopy equivalences $[\mathscr{N}, \mathscr{A}]_{\text {h.e. }}$. This follows from the fact that any homotopy equivalence $\beta: \mathscr{N} \rightarrow \mathscr{A}$ is injective, since its projection $\pi \circ \beta: \mathscr{N} \rightarrow \mathscr{A} \rightarrow \mathscr{N}$ is an isomorphism.

The maps $\bar{\pi}$ and $\bar{j}$ are isomorphisms because $\pi$ and $j$ are homotopy equivalences.

The map $h_{2}$ is surjective as composition of surjective maps.

Surjectivity of $h_{1}$ : Let $\alpha: \mathscr{A} \rightarrow \mathscr{A}$ be a homotopy equivalent such that $\alpha \circ i \simeq i$. By successive application of Proposition 2.6 we can extend $i$ to a cohomology isomorphism $\alpha^{\prime}: \mathscr{A} \rightarrow \mathscr{A}$

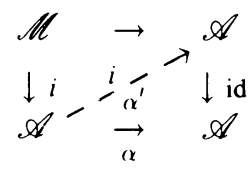

But by Theorem 2.17, $\alpha^{\prime}$ is actually an isomorphism.

By a similar argument we can show that the composition $\bar{\pi} \circ \bar{j} \circ h_{1}$ : $\operatorname{Aut}(\mathscr{A}, \operatorname{rel} \mathscr{M}) \rightarrow \operatorname{aut}(\mathscr{N})_{[\varphi]}$ is surjective. Let $\alpha: \mathscr{N} \rightarrow \mathscr{N}$ be an isomorphism 
which preserves $\varphi$ up to homotopy. We consider the diagram

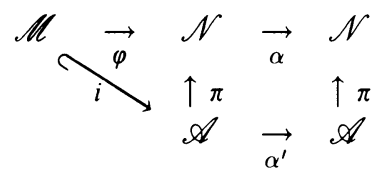

and define $\alpha^{\prime} \mid \mathscr{M}=\mathrm{id}_{\mathscr{M}}$, where $\mathscr{M}$ is identified with its image $i(\mathscr{M})$ in $\mathscr{A}$. One can check easily that $\pi \circ \alpha^{\prime}|\mathscr{M} \simeq \alpha \circ \pi| \mathscr{M}$. As above we extend $\alpha^{\prime} \mid \mathscr{M}$ to an isomorphism $\alpha^{\prime}: \mathscr{A} \rightarrow \mathscr{A}$.

All maps of the first row are algebraic maps between algebraic varieties. The elements of these algebraic varieties are matrices over $\mathbf{Q}$ and the maps between them are matrix multiplications. Also the map $h_{4}$ is an algebraic epimorphism between algebraic groups by [S2]. Then aut $\mathscr{A})$ can be given the structure of an algebraic matrix group over $\mathbf{Q}$ via the isomorphism $\bar{\pi} \circ \bar{j}$, and the map $h_{2}$ becomes a map of algebraic groups. It is easy to see that $\operatorname{Aut}(\mathscr{A}, \operatorname{rel} \mathscr{M})$ is an algebraic subgroup of $\operatorname{Aut}(\mathscr{A})$. If we think of the elements of $\operatorname{Aut}(\mathscr{A})$ as matrices then $\operatorname{Aut}(\mathscr{A}, \operatorname{rel} \mathscr{M})$ contains the matrices $\operatorname{Aut}(\mathscr{A})$ which are of the form $\left(\begin{array}{cc}\text { id } & A \\ 0 & B\end{array}\right)$, i.e. they satisfy linear equations on the entries.

Since the map $\bar{\pi} \circ \bar{j} \circ h_{1}=\bar{\pi} \circ \bar{j} \circ h_{2} \mid \operatorname{Aut}(\mathscr{A}$, rel $\mathscr{M})$ is an algebraic group homomorphism, its image $\operatorname{aut}(\mathscr{N})_{[\varphi]}$ is an algebraic subgroup of $\operatorname{aut}(\mathscr{N})$. This completes the proof of Theorem 1.2(i).

In order to prove Theorem 1.2(ii), we will use the following result about principal fibrations.

Theorem 3.1. Let $F \rightarrow E \stackrel{p}{\rightarrow} B$ be a principal fibration, where $F=K(\pi, n)$, and $\pi$ is finitely generated. Let $X$ be a finite nilpotent $C W$-complex and let $f^{\prime}: X \rightarrow E$ and $f: X \rightarrow B$ be maps such that $p \circ f^{\prime}=f$. Assume that $\operatorname{aut}(X)_{[f]}$ is commensurable to an arithmetic subgroup of $\operatorname{aut}\left(X_{0}\right)_{\left[f_{0}\right]}$. Then $\operatorname{aut}(X)_{\left[f^{\prime}\right]}$ is commensurable to an arithmetic subgroup of aut $\left(X_{0}\right)_{\left[f_{0}^{\prime}\right]} \subset \operatorname{aut}\left(X_{0}\right)_{\left[f_{0}\right]}$.

Proof. Let $\rho: \mathscr{M} \rightarrow A(B) \rightarrow \mathscr{A}\left(B_{0}\right)$ be a minimal model for $B$ and $B_{0}$, and let $\sigma: \mathscr{N} \rightarrow A(X) \rightarrow A\left(X_{0}\right)$ be a minimal model for $X$ and $X_{0}$. By Theorem 2.20, a minimal model of $E$ is an elementary extension of $\mathscr{M}$, namely $\mathscr{M} \otimes_{d} \Lambda V$, where $V \cong \pi^{*}$ and $d: V \rightarrow Z^{n+1}(\mathscr{M})$ is a representative of the classifying cohomology class. Call $\mathscr{M}^{\prime}=\mathscr{M} \otimes_{d} \Lambda V$ and let $\rho^{\prime}: \mathscr{M}^{\prime} \rightarrow A(E)$ be the cohomology isomorphism which extends $\rho$.

Let $\varphi: \mathscr{M} \rightarrow \mathscr{N}$ be a map between the minimal models induced by $f$ and let $\varphi^{\prime}: \mathscr{M}^{\prime} \rightarrow \mathscr{N}$ be an extension of $\varphi$ induced by $f^{\prime}$. Let $i^{\prime}: \mathscr{M}^{\prime} \rightarrow \mathscr{A}$ be a minimal extension for $\varphi^{\prime}$ and let $\pi^{\prime}: \mathscr{A} \rightarrow \mathscr{N}$ be a cohomology isomorphism such that the diagram

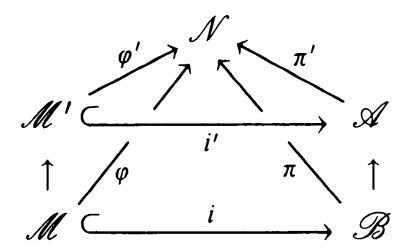

commutes. Now $i^{\prime} \mid \mathscr{M}$ is a free extension for $\varphi$ and therefore, by [H, Theorem 2.2], it factors through a minimal extension $i: \mathscr{M} \hookrightarrow \mathscr{B}$, such that $\mathscr{A}=\mathscr{B} \otimes \mathscr{R}$ and $\mathscr{R}$ is a free acyclic dga. Both $\mathscr{A}$ and $\mathscr{B}$ have the same homotopy type as $\mathscr{N}$. 
Consider now the following commutative diagram

$$
\begin{aligned}
& \operatorname{Map}\left(V, Z^{n}(\mathscr{A})\right) \rightarrow H^{n}(\mathscr{A}, V) \stackrel{\downarrow}{\cong} H^{n}(\mathscr{N}, V) \cong H^{n}(X, \pi \otimes \mathbf{Q}) \leftarrow H^{n}(X, \pi)
\end{aligned}
$$

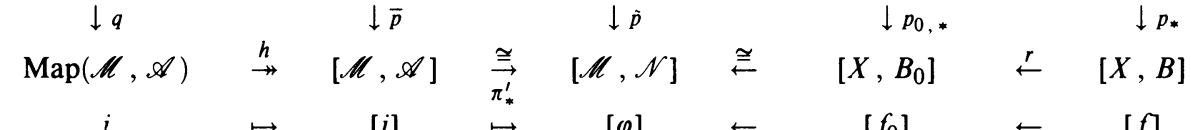

where the top vertical arrows mean actions of groups on the sets of the second row, $\operatorname{Map}(\mathscr{M}, \mathscr{A})$ is the set of dga maps from $\mathscr{M}$ to $\mathscr{A}, h$ means projection to homotopy classes and $r$ means rationalization. The set $Z^{n}(\mathscr{A})$ contains the cocylces of $\mathscr{A}$ of degree $n$ and $\operatorname{Map}\left(V, Z^{n}(\mathscr{A})\right)$ means linear maps of vector spaces.

The vector space $\operatorname{Map}\left(V, Z^{n}(\mathscr{A})\right)$ acts on $\operatorname{Map}\left(\mathscr{M}^{\prime}, \mathscr{A}\right)$ as follows: Let $\tau: V \rightarrow Z^{n}(\mathscr{A})$ and let $\psi: \mathscr{M}^{\prime} \rightarrow \mathscr{A}$. Define $\tau \psi: \mathscr{M}^{\prime} \rightarrow \mathscr{A}$ to be the dga map which is equal to $\psi$ when restricted to $\mathscr{M} \subset \mathscr{M}^{\prime}$ and is equal to $\psi+\tau$ when restricted to $V$. By [G-M, p. 178] this action induces the action of $H^{n}(\mathscr{A}, V)$ on $\left[\mathscr{M}^{\prime}, \mathscr{A}\right]$ which is compatible with the action of $H^{n}(X, \pi)$ on $[X, E]$.

Consider all homotopy classes of maps from $\mathscr{M}^{\prime}$ to $\mathscr{A}$ the restriction of which on $\mathscr{M} \subset \mathscr{M}^{\prime}$ are homotopic to $i$, i.e., consider the set $\bar{p}^{-1}([i]) \subseteq$ $\left[\mathscr{M}^{\prime}, \mathscr{A}\right]$. Also consider the set of extensions of $i, q^{-1}(i) \subseteq \operatorname{Map}\left(\mathscr{M}^{\prime}, \mathscr{A}\right)$.

Claim. $h \mid q^{-1}(i)$ is a surjection onto $\bar{p}^{-1}([i])$.

This follows from the homotopy extension property of elementary extensions (Proposition 2.6).

The vector space $\operatorname{Map}\left(V, Z^{n}(\mathscr{A})\right)$ acts freely and transitively on $q^{-1}(i)$ and therefore it can be identified with $q^{-1}(i)$ via the map

$$
\begin{aligned}
q^{-1}(i) & \rightarrow \operatorname{Map}\left(V, Z^{n}(\mathscr{A})\right) \\
\psi & \mapsto \psi-i^{\prime},
\end{aligned}
$$

where $i^{\prime}: \mathscr{M}^{\prime} \hookrightarrow \mathscr{A}$ is the minimal extension for $\varphi^{\prime}$ as described above.

Now we restrict attention to a piece of diagram (3.2), namely

$$
\begin{array}{ccc}
\operatorname{Map}\left(V, Z^{n}(\mathscr{A})\right) & \rightarrow & H^{n}(\mathscr{A}, V) \\
t \downarrow \cong & & \downarrow s \\
q^{-1}(i) & \rightarrow & \bar{p}^{-1}([i]),
\end{array}
$$

where $s$ is induced by $t$, i.e., it is the projection onto the orbit of $\left[i^{\prime}\right]$. The kernel of $s$, say $C=\operatorname{ker} s$, is a subvector space over $\mathbf{Q}$ of $H^{n}(\mathscr{A}, V)$, and it is described algebraically in [G-M]. Geometrically, it corresponds to the image of a group homomorphism

$$
c: \pi_{1}\left(B^{X}, f\right) \rightarrow H^{n}(X, \pi) .
$$

This map is the composite of $k_{*}: \pi_{1}\left(B^{X}, f\right) \rightarrow \pi_{1}\left(K(\pi, n+1)^{X}, k \circ f\right)$, where $k: B \rightarrow K(\pi, n+1)$ is the classifying map of the fibration, and a change of base point.

$$
\pi_{1}\left(K(\pi, n+1)^{X}, k \circ f\right) \stackrel{\cong}{\rightrightarrows} \pi_{1}\left(K(\pi, n+1)^{X}, *\right)=[X, \Omega K(\pi, n+1)]
$$

(cf. [R, p. 382]). 
By results of [B-K and H-M-R] on localization of function spaces, $\pi_{1}\left(B^{X}, f\right)$ is nilpotent, and after rationalizing $B$

$$
c\left(\pi_{1}\left(B^{X}, f\right)\right) \otimes \mathbf{Q} \cong c\left(\pi_{1}\left(B_{0}^{X}, f_{0}\right)\right) \subseteq H^{n}(X ; \pi \otimes \mathbf{Q}) .
$$

On the other hand, $c\left(\pi_{1}\left(B_{0}^{X}, f_{0}\right)\right) \cong C \equiv \operatorname{ker} s$.

Now $\operatorname{Aut}(\mathscr{A}, \operatorname{rel} \mathscr{M})$ acts on $q^{-1}(i)$ by composition, i.e., $\alpha(g)=\alpha \circ g$, and therefore it acts on $\operatorname{Map}\left(V, Z^{n}(\mathscr{A})\right)$ via the above identification by

$$
\alpha(\tau)=\alpha \circ \tau+\left(\alpha \circ i^{\prime}-i^{\prime}\right)
$$

here $\tau: V \rightarrow Z^{n}(\mathscr{A}), \alpha: \mathscr{A} \rightarrow \mathscr{A}$ is an automorphism which restricts to the identity on $\mathscr{M} \hookrightarrow \mathscr{A}$, and $i^{\prime}: \mathscr{M}^{\prime} \rightarrow \mathscr{A}$ is the minimal extension given above.

This is an algebraic action by affine transformations on the vector space $\operatorname{Map}\left(V, Z^{n}(\mathscr{A})\right)$, namely the action by $\alpha$ is the composition of a linear automorphism of $\operatorname{Map}\left(V, Z^{n}(\mathscr{A})\right)$ on the one hand and a translation by $\alpha \circ i^{\prime}-i^{\prime}$ on the other. To see that it is an algebraic action, consider $\tau, \alpha$, and $i$ as matrices. Then $\alpha(\tau)$ is a matrix the entries of which are polynomial, in fact linear, expressions of the entries of $\tau$. Let $U \equiv \operatorname{Map}\left(V, Z^{n}(\mathscr{A})\right)$ for brevity. Then we have an algebraic group homomorphism

$$
\phi: \operatorname{Aut}(\mathscr{A}, \operatorname{rel} \mathscr{M}) \rightarrow \operatorname{Aff}(U) \cong U \ltimes \mathrm{GL}(U)
$$

We remark that $\operatorname{Aff}(U)$ can be identified with an algebraic subgroup of $\mathrm{GL}(U \oplus \mathbf{Q})$ via the correspondence

$$
\begin{gathered}
U \ltimes \mathrm{GL}(U) \rightarrow \mathrm{GL}(U \oplus \mathbf{Q}) \\
(u, g) \mapsto\left(\begin{array}{cc}
g & u \\
0 & 1
\end{array}\right) .
\end{gathered}
$$

Now let $\operatorname{aut}(\mathscr{A}, \widetilde{\operatorname{rel}} \mathscr{M})$ be the group of homotopy classes of self homotopy equivalences of $\mathscr{A}$ which are homotopic to $\mathrm{id}_{\mathscr{M}}$ when restricted to $\mathscr{M} \hookrightarrow \mathscr{A}$. The action of $\operatorname{Aut}(\mathscr{A}$, rel $\mathscr{M})$ on $q^{-1}(i)$ induces an action of $\operatorname{aut}(\mathscr{A}, \widetilde{\operatorname{rel}} \mathscr{M})$ on $\bar{p}^{-1}([i]) \cong H^{n}(\mathscr{A}, V) / C$ (cf. diagram (3.3)). We call $H^{n}(\mathscr{A}, V) / C \equiv W$ and we let $\theta^{\prime}: \operatorname{aut}(\mathscr{A}, \widetilde{\operatorname{rel}} \mathscr{M}) \rightarrow \operatorname{Aff}(W)$ be the induced action. Then we have a commutative diagram

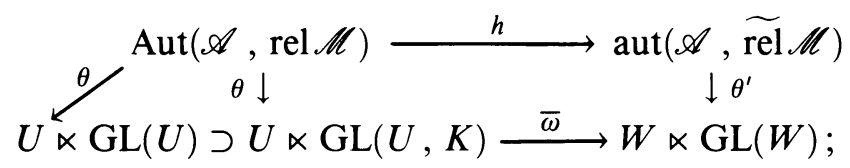

here $K$ is the kernel of

$$
\omega: U \equiv \operatorname{Map}\left(V, Z^{n}(\mathscr{A})\right) \rightarrow H^{n}(\mathscr{A}, V) / C \equiv W,
$$

$\mathrm{GL}(U, K) \subseteq \mathrm{GL}(U)$ is the subgroup of linear automorphisms of $U$ which map $K$ to itself, and $\bar{\omega}$ is the map induced by $\omega: U \rightarrow W$.

It has been shown in earlier pages that $h$ is an algebraic group epimorphism. The map $\bar{\omega} \circ \theta$ is obviously an algebraic homomorphism too. Therefore, $\theta^{\prime}$ is an algebraic homomorphism as well. This follows from the factorization property of algebraic group homomorphisms when we divide by algebraic normal subgroups (cf. [Hu, p. 83]). 
In conclusion, we have an algebraic action of $\operatorname{aut}(\mathscr{A}, \widetilde{\operatorname{rel}} \mathscr{M})$ on $H^{n}(\mathscr{A}, V) / C$ $=\bar{p}^{-1}([i])$, or isomorphically, we have an algebraic action of $\operatorname{aut}(\mathscr{N})_{[\varphi]}$ on $H^{n}(\mathcal{N}, V) / C=\tilde{p}^{-1}([\varphi])$, which we call again $W$.

Relating the action $\theta^{\prime}$ to the topological action we have a commutative diagram

$$
\begin{array}{ccc}
\operatorname{aut}(\mathscr{M})_{[\varphi]} & \rightarrow & \operatorname{Aff}(W)) \\
\uparrow & & \uparrow \\
\operatorname{aut}(X)_{[f]} & \rightarrow & \operatorname{Aff}(\Gamma),
\end{array}
$$

where $\Gamma=H^{n}(X, \pi) / c\left(\pi_{1}\left(B^{X}, f\right)\right)$. The image of $\Gamma$ in $W$ is a lattice of $W$ and the kernel of $\Gamma \rightarrow W$ is finite. Since $\Gamma=H^{n+1}(X, \pi) / c\left(\pi_{1}\left(B^{X}, f\right)\right)=$ $p_{*}^{-1}([f])$ (cf. diagram (3.2)), i.e., it classifies homotopy classes of liftings of $f$, $\Gamma$ contains $\left[f^{\prime}\right]$. We observe that the isotropy group of $\left[f^{\prime}\right] \in \Gamma$ under the action of $\operatorname{aut}(X)_{[f]}$ is equal to $\operatorname{aut}(X)_{\left[f^{\prime}\right]}$, and the isotropy group of $\left[\varphi^{\prime}\right] \in W$ under the action of $\operatorname{aut}(\mathscr{N})_{[\varphi]}$ is equal to $\operatorname{aut}(\mathscr{N})_{\left[\varphi^{\prime}\right]}$. By assumption $\operatorname{aut}(X)_{[\varphi]}$ is commensurable to an arithmetic subgroup of $\operatorname{aut}(\mathscr{N})_{[\varphi]} \cong \operatorname{aut}\left(X_{0}\right)_{\left[f_{0}\right]}$. As we explain below, when we have an algebraic action of $G$ on a vector space $W$ and $A \subset G$ is an arithmetic subgroup of $G$ then (a) the isotropy group $G_{w} \subset G$ of an element $w \in W$ is algebraic and (b) $A_{w}$ is an arithmetic subgroup of $G_{w}$. Therefore $\operatorname{aut}(X)_{\left[f^{\prime}\right]}$ is commensurable to an arithmetic subgroup of $\operatorname{aut}(\mathcal{N})_{\left[\varphi^{\prime}\right]} \cong \operatorname{aut}\left(X_{0}\right)_{\left[f_{0}^{\prime}\right]}$. This completes the proof of Theorem 3.1, modulo (a) and (b).

Now let $\theta: G \rightarrow \mathrm{GL}(W)$ be an algebraic action of $G$ on $W$, and let $A \subset G$ be an arithmetic subgroup. Statement (a) above is obvious since $G_{w}$ is defined by polynomial, in fact linear equations $\theta(g) w=w$ on the entries of $g$. Now $G$ is by definition a subgroup of some $\operatorname{GL}(V)$ satisfying a set of polynomial equations. Let $G(L) \subset G$ be the arithmetic subgroup determined by a lattice $L$. Then $G(L)_{w}$ is obviously an arithmetic subgroup of $G_{w}$. If $A \subset G(L)$ is a subgroup of finite index in $G(L)$ then $A_{w}=A \cap G(L)_{w}$ is a subgroup of finite index in $G(L)_{w}$. If $A$ is an arbitrary arithmetic subgroup of $G$ then $A \cap G(L)$ has finite index in $A$ and in $G(L)$. In this case we apply the previous statement twice to get that $A_{w}$ is commensurable to $G(L)_{w}$, i.e., $A_{w}$ is an arithmetic subgroup of $G_{w}$.

Proof of Theorem 1.2(ii). The proof can be done inductively over the Postnikov tower of principal fibrations of $Y$ starting from a point and using Theorem 3.1 as the inductive step. The assumption that $X$ is a finite $C W$-complex guarantees that the process stops since $[X, Y]=\left[X, Y_{n}\right]$ for some big enough $n$.

\section{REFERENCES}

[B-G] A. K. Bousfield and V. K. A. M. Gugenheim, On PL de Rham theory and rational homotopy type, Mem. Amer. Math. Soc., vol. 8, no. 179, 1976.

[B-HC] A. Borel and Harish-Chandra, Arithmetic subgroups of algebraic groups, Ann. of Math. (2) 75 (1962), 485-535.

[B-K] A. K. Bousfield and D. M. Kan, Homotopy limits, completions and localizations, Lecture Notes in Math., vol. 304, Springer-Verlag, 1972.

[B-S] A. Borel and J. P. Serre, Corners and arithmetic groups, Comment. Math. Helv. 48 (1973), 436-491. 
[D-D-K] E. Dror, W. C. Dwyer and D. M. Kan, Self homotopy equivalences of virtually nilpotent spaces, Comment. Math. Helv. 56 (1981), 599-614.

[G-M] P. A. Griffiths and J. W. Morgan, Rational homotopy theory and differential forms, Birkhäuser Verlag, Basel, 1981.

[H] S. Halperin, Lectures on minimal models, Mem. Soc. Math. France 9 (1983).

[H-M-R] P. J. Hilton, G. Mislin and J. Roitberg, Localization of nilpotent groups and spaces, NorthHolland Math. Studies, no. 15, North-Holland, 1975.

[Hr] S. Hurwitz, The automorphism groups of spaces and fibrations, Pacific J. Math. 96 (1981), 371-388.

[Hu] J. E. Humphreys, Linear algebraic groups, Graduate Texts in Math., vol. 21, SpringerVerlag, 1975.

[Q] D. Quillen, Rational homotopy theory, Ann. of Math. (2) 90 (1969), 205-295.

[R] J. W. Rutter, A homotopy classification of maps into an induced fibre space, Topology 6 (1967), 379-403.

[S1] D. Sullivan, Geometric topology, part I: Localization, periodicity and Galois symmetry, M.I.T. Press, Cambridge, Mass., 1970.

[S2] _ Infinitesimal computations in topology, Publ. Math. Inst. Hautes Études Sci. 47 (1978), 269-331.

[W] C. W. Wilkerson, Minimal simplicial groups, Topology 15 (1976), 111-130.

Department of Mathematics, University of Crete, Crete, Greece

Current address: Department of Mathematics, University of Chicago, Chicago, Illinois 60637

E-mail address: georgia@zaphod.uchicago.edu 